\title{
sE-cadherin serves as a diagnostic and predictive parameter in prostate cancer patients
}

Igor Tsaur ${ }^{1 *}$ (D, Kristina Thurn ${ }^{1}$, Eva Juengel ${ }^{1}$, Kilian M. Gust $^{1}$, Hendrik Borgmann ${ }^{1}$, Rene Mager ${ }^{1}$, Georg Bartsch', Elsie Oppermann ${ }^{2}$, Hanns Ackermann ${ }^{3}$, Karen Nelson ${ }^{4}$, Axel Haferkamp ${ }^{1}$ and Roman A. Blaheta ${ }^{1}$

\begin{abstract}
Background: Measurement of prostate-specific antigen (PSA) advanced the diagnostic and prognostic potential for prostate cancer (PCa). However, due to PSA's lack of specificity, novel biomarkers are needed to improve risk assessment and ensure optimal personalized therapy. A set of protein molecules as potential biomarkers was therefore evaluated in serum of PCa patients.

Methods: Serum samples from patients undergoing radical prostatectomy (RPE) for biopsy-proven PCa without neoadjuvant treatment were compared to serum samples from healthy subjects. Preliminary screening of 119 proteins in 10 PCa patients and 10 controls was carried out by the Proteome Profiler Antibody Array. Those markers showing distinct differences between patients and controls were then further evaluated by ELISA in the serum of 165 PCa patients and 19 controls. Uni- and multivariate as well as correlation analysis were performed to test the capability of these molecules to detect disease and predict pathological outcome.

Results: Screening showed that soluble (s)E-cadherin, E-selectin, MMP2, MMP9, TIMP1, TIMP2, Galectin and Clusterin warranted further evaluation. sE-Cadherin, TIMP1, Galectin and Clusterin were significantly over- and MMP9 under-expressed in PCa compared to controls. The concentration of SE-cadherin, MMP2 and Clusterin correlated negatively and that of MMP9 and TIMP1 positively with the Gleason Sum at prostatectomy. Only sE-cadherin significantly correlated with the highest Gleason pattern. Compared to serum PSA, sE-cadherin provided an independent and better matching predictive ability for discriminating PCas with an upgrade at RPE and aggressive tumors with a Gleason Sum $\geq 7$.

Conclusions: sE-cadherin performed most favorably from a large panel of serum proteins in terms of diagnostic and predictive potential in curatively treatable PCa. SE-cadherin merits further investigation as a biomarker for PCa.
\end{abstract}

Keywords: Prostate cancer, Diagnosis, Prediction, Biomarker, sE-cadherin

\section{Background}

Prostate cancer $(\mathrm{PCa})$ is currently the most prevalent male neoplasia in industrialized countries, accounting for almost $12 \%$ of cancer cases annually [1]. Besides incumbent morbidity, the disease is associated with a significant economic burden. Declining mortality is expected to cause increases in healthcare costs as a result of increased diagnosis, diagnosis at an earlier stage and increased survival [2].

\footnotetext{
* Correspondence: igor.tsaur@kgu.de

'Department of Urology, Goethe-University, Frankfurt am Main, Germany Full list of author information is available at the end of the article
}

Due to the biological heterogeneity of PCa and rapidly expanding treatment options, individualized risk-adapted therapy incorporating patient- and tumor-specific characteristics is required to optimize outcome and avoid overtreatment with unnecessary adverse effects [3, 4]. Since not only radical prostatectomy (RPE) or radiation, but also active surveillance possibly postponing definite therapy, currently represent state-of-the-art care for patients with a localized PCa and a long life expectancy [5], identifying cancer types with high progression risk is indispensable in determining a treatment course. Nomograms, neural networks and predictive tables have all been developed in the 
past years to tailor decision-making at different stages of PCa diagnosis and treatment [6]. Being limited in their clinical application by problematic generalization and population-specific issues [7], they provide an average accuracy of $70 \%$ [6]. To improve their performance, identification of appropriate molecular biomarkers and possibly their incorporation into predictive models is crucial [7].

Until now, prostate specific antigen (PSA) remains the only clinically relevant diagnostic and follow-up biomarker [8]. Recent research has been aimed at finding markers to overcome the limits of PSA, not only to diagnose PCa but also to distinguish between indolent and clinically significant disease [9]. Despite initial promising reports, these marker-candidates have fallen short following extensive validation and have not proven to be of routine value.

Marker detection in serum is attractive since sampling is simple and ideal for screening and prognostic evaluation of PCa to facilitate treatment choices for patients with local disease [10]. A panel of serum candidate markers known to be involved in inflammatory processes and tumorigenesis of different cancer types was evaluated for diagnostic and predictive potential in a cohort of patients with PCa.

\section{Methods}

\section{Patients and controls}

Study approval was granted by the local medical ethics committee (project number SUG_01-2014). Patients $(\mathrm{n}=165)$ undergoing curative radical prostatectomy for biopsy-proven $\mathrm{PCa}$ without any neoadjuvant treatment between October 2010 and June 2014 in the Department of Urology, Goethe-University, Frankfurt am Main, Germany, were included in the study after having signed informed consent to utilize biomaterial for scientific assessment. The clinical tumor stage was classified according to the 7th edition of AJCC [11] and the pathological tumor stage was determined according to the 6th edition of the TNM classification [12]. Tumors were graded with the Gleason Sum (GS) [13]. Clinical and histological characteristics were collected from patient charts. Risk classification was determined according to D'Amico et al. [14]. Imaging was carried out in the high-risk group according to the guidelines of the European Association of Urology [15]. Epstein criteria [16] were used to assess the clinical significance of the tumor. Controls $(n=19)$ were healthy, age-matched, male volunteers.

\section{Blood samples}

$10 \mathrm{ml}$ peripheral blood was drawn from patients several days before surgery and from controls. Blood samples were allowed to coagulate and then centrifuged at $3000 \mathrm{rpm}$ at $+4{ }^{\circ} \mathrm{C}$ for $10 \mathrm{~min}$. The serum supernatant was stored at $-80{ }^{\circ} \mathrm{C}$.

\section{Proteome profiler antibody array}

Serum from 10 PCa patients and from 10 controls were applied to a proteome profiler array to screen 119 markers (Human soluble Receptor Array Kit, Non Hematopoietic Panel, R\&D systems, MN, USA) (Table 1) according to the manufacturer's instructions. Briefly, membranes spotted with antibodies were blocked with Array buffer for $1 \mathrm{~h}$ at room temperature on a shaker followed by incubation with $1 \mathrm{ml}$ serum mixed with $3 \mathrm{ml}$ of array buffer overnight at $4{ }^{\circ} \mathrm{C}$. On the next day membranes were washed $3 \mathrm{x}$ with wash buffer and further incubated with a detection antibody cocktail for $2 \mathrm{~h}$ at room temperature on a shaker. Membranes were again washed $3 x$ with wash buffer and incubated with Streptavidin-HRP for $30 \mathrm{~min}$ at room temperature on a shaker. Signals were visualized using the Chemi reagent mix on a FUSION FX-7 imaging device (Vilber Lourmat, Torcy, France).

\section{Elisa}

E-selectin, soluble (s)E-Cadherin, MMP2, MMP9, TIMP1, TIMP2, Galectin and Clusterin, were identified as distinctly differently expressed in the serum of PCa patients and controls in the Proteome Profiler Antibody Array screening. The concentration of these markers was determined in the serum of 165 PCa patients and 19 controls using commercially available ELISA Kits (R\&DSystems, MN, USA) according to the manufacturer's instructions. Briefly, serum was diluted, pipetted into wells and incubated at $37{ }^{\circ} \mathrm{C}$ or RT for 1-2 $\mathrm{h}$ according to kit instructions. After incubation plates were washed $4 \mathrm{x}$, followed by incubation with detection antibodies for $30 \mathrm{~min}$ or $1 \mathrm{~h}$ at RT and wells were subsequently washed $4 \mathrm{x}$. TMB substrate was added to each well for $20 \mathrm{~min}$ at RT, followed by the addition of stop solution. The optical density was measured spectrophotometrically with an ELISA reader (Infinite M200 series, Tecan, Crailsheim, Germany). All assays were done in duplicate and the concentration was calculated from a standard curve using a 4-parameter curve fit (Magellan software, Tecan).

\section{Statistics}

Univariate analysis was performed by the WilcoxonMan-Whitney-test for comparison between two groups and the Kruskal-Wallis-test with the Iman-Conovermethod (Bonferroni-Holm-corrected) for more than two groups. Bi- and multivariate analysis was carried out by logistic regression for two target values and multiple regression for more than two target values. Correlation between two parameters was evaluated through Spearman's coefficient. The statistical program applied was BiASfuer Windows (Version 9.11, Dr. rer. nat. Hanns Ackermann, epsilon-publishers, Frankfurt, Germany). The null hypothesis was rejected if $\mathrm{p}$-values were less than 0.05 . Continuous data were presented as median (range) or number (\%), as applicable. 
Table 1 Markers screened with Proteome Profiling Antibody

\begin{tabular}{|c|c|c|c|c|}
\hline \multicolumn{5}{|l|}{ A: Non-Hematopoietic Array } \\
\hline ADAM15 & $\beta \mid G-H 3$ & BMPR-IB/ALK-6 & Cadherin-4/R-Cadherin & Cadherin-11 \\
\hline Cadherin-13 & E-Cadherin & $\mathrm{N}$-Cadherin & P-Cadherin & VE-Cadherin \\
\hline Cathepsin-D & CD40/TNFRSF5 & CEACAM-5/CD66e & CHL-1/L1CAM-2 & Clusterin \\
\hline Coagulation-Factor II/Thrombin & COMP/Thrombospondin & CRELD2 & Desmoglein 2 & ECM-1 \\
\hline EGF R/ErbB1 & Endoglycan & EpCAM/TROP-1 & ErbB2/HER2 & ErbB3/HER3 \\
\hline ErbB4/HER4 & ESAM & Galectin-2 & HPRG & Integrin a3/CD49c \\
\hline Integrin a5/CD49e & Integrin a6/CD49f & Integrin a9 & Integrin aV/CD51 & Jagged 1 \\
\hline JAM-B/VE-JAM & JAM-C & LRP-6 & MCAM/CD146 & MEPE \\
\hline MUCDHL & Nectin-2/CD112 & Nectin-4 & Neurotrimin & Notch-1 \\
\hline NrCAM & Periostin/OSF-2 & Podocalyxin & E-Selectin/CD62e & Semaphorin-3A \\
\hline SREC-I/SR-F1 & SREC-II & Stanniocalcin 1 & Syndecan-1/CD138 & Syndecan-4 \\
\hline Thrombospondin-2 & TIMP-4 & TROP-2 & VAP-1/AOC3 & VCAM-1 \\
\hline VEGF R1/Flt-1 & VEGF R2/KDR/Flk-1 & & & \\
\hline \multicolumn{5}{|l|}{ B: Common analytes array } \\
\hline ACE & ADAM8 & ADAM9 & ADAM10 & ALCAM/CD166 \\
\hline Amphiregulin & APP (pan) & BACE-1 & BCAM & C1qR1/CD93 \\
\hline CD9 & CD23/Fc \& RII & CD31/PECAM-1 & CD36/SR-B3 & CD40 Ligand/TNFSF5 \\
\hline $\mathrm{CD} 44 \mathrm{H}$ & CD58/LFA-3 & CD90/Thy1 & CD99 & CD155/PVR \\
\hline CEACAM-1/CD66a & CX3CL1/Fractalkine & CXCL8/IL-8 & EMMPRINN/CD147 & Endoglin/CD105 \\
\hline Epiregulin & Galectin-1 & Galectin-3 & Galectin-3BP/MAC-2BP & HB-EGF \\
\hline ICAM-2/CD102 & $\| \mathrm{L}-1 \mathrm{R \|}$ & IL-15 Ra & Integrin $\beta 1 / C D 29$ & Integrin $\beta 2 / C D 18$ \\
\hline Integrin $\beta 3 / C D 61$ & Integrin $\beta 4 / C D 104$ & Integrin $\beta 5$ & Integrin $\beta 6$ & JAM-A \\
\hline Lipoclain-2/NGAL & LOX-1/SR-E1 & MD-1 & MMP-2 (total) & NCAM-1/CD56 \\
\hline NCAM-L1 & Osteopontin & PAR1 & Pref-1/DLK-1/FA1 & RECK \\
\hline Stabilin-1 & TACE/ADAM17 & Thrombospondin & TIMP-1 & TIMP-2 \\
\hline TIMP-3 & TNF RII/TNFRSF1B & & & \\
\hline
\end{tabular}

\section{Results}

Clinical and pathologic demographics of 165 patients are shown in Table 2. The median age at tumor diagnosis was 65 years and the median serum PSA was $8.1 \mathrm{ng} / \mathrm{ml}$. Nearly all PCas submitted to surgery were clinically significant. All patients were clinically free of visceral or bone disease. None of the patients had evident clinical signs of infection or acute or chronic inflammation at surgery. Histologically, all tumors were conventional acinar adenocarcinomas.

The screening analysis by Proteome Profiler showed distinctly altered expression of sE-cadherin, E-selectin, MMP2, MMP9, TIMP1, TIMP2, Galectin and Clusterin in the serum of tumor patients compared to controls. Further ELISA investigations were based on these markers.

Univariate analysis of the ELISA investigation demonstrated that serum sE-Cadherin, TIMP1, Galectin and Clusterin were significantly over- and MMP9 underexpressed in $\mathrm{PCa}$ patients compared to healthy controls (all $\mathrm{p}<0.05$, Fig. 1). The concentration of sE-Cadherin,
MMP2 and Clusterin correlated negatively and that of MMP9 and TIMP1 positively with GS at prostatectomy (all $\mathrm{p}<0.05$, Table 3 ), but only $\mathrm{sE-cadherin} \mathrm{significantly}$ correlated with the highest Gleason pattern $(\mathrm{p}<0.05$, Fig. 2a). In the group of patients with a GS upgrade from prostate biopsy to prostatectomy specimen, a decreased concentration of sE-cadherin was observed, compared to patients without upgrade ( $\mathrm{p}<0.05$, Fig. $2 \mathrm{~b})$.

Multivariate analysis with logistic regression for all 8 candidate markers as well as serum PSA was unsuitable because, taking into account the cohort size and the rule of ten' [17], sufficient stability of the model could not be ensured. Therefore, a bivariate logistic regression analysis was performed, incorporating each of the candidate molecules and serum PSA to assess their ancillary predictive potential over PSA for clinical and pathological T-stage, L-, N-, R-, Pn-status, biopsy (b) and prostatectomy $(\mathrm{p}) \mathrm{GS}$ after dichotomization of the study cohort into $\mathrm{GS}<7$ and $\geq 7$, as well as Gleason upgrade. Only sE-cadherin yielded favorable discriminative ability 
Table 2 Clinical and histopathological demographics of 165 PCa patients

\begin{tabular}{|c|c|}
\hline & $n=165(100 \%)$ \\
\hline Age, yrs & 65 (range: 40-88) \\
\hline \multicolumn{2}{|l|}{ pT-stage } \\
\hline$<3$ & $113(68.5)$ \\
\hline$\geq 3$ & $52(31.5)$ \\
\hline Extracapsular infiltration & $34(20.6)$ \\
\hline Infiltration of seminal vesicles & $18(11.0)$ \\
\hline \multicolumn{2}{|l|}{ cT-stage } \\
\hline$<3$ & $158(95.8)$ \\
\hline$\geq 3$ & $7(4.2)$ \\
\hline Serum-PSA, ng/ml & $8.1(1.8-136)$ \\
\hline PSAD, ng/ml/ml & $0.2(0.1-1.8)$ \\
\hline Abnormal DRE & $65(39.4)$ \\
\hline Prostate volume, ml & $33(10-120)$ \\
\hline \multicolumn{2}{|l|}{ Gleason Sum (Biopsy) } \\
\hline$\leq 6$ & $86(52.1)$ \\
\hline 7 & $55(33.3)$ \\
\hline$\geq 8$ & $24(14.5)$ \\
\hline \multicolumn{2}{|l|}{ Highest Gleason Pattern (Biopsy) } \\
\hline 3 & $70(42.4)$ \\
\hline 4 & $62(37.6)$ \\
\hline 5 & $14(8.5)$ \\
\hline \multicolumn{2}{|l|}{ Gleason Sum (RPE) } \\
\hline 6 & $27(16.4)$ \\
\hline 7 & $99(60.0)$ \\
\hline$\geq 8$ & 39 (23.6) \\
\hline \multicolumn{2}{|l|}{ Highest Gleason Pattern (RPE) } \\
\hline 3 & $28(17.0)$ \\
\hline 4 & $104(63.0)$ \\
\hline 5 & $33(20.0)$ \\
\hline \multicolumn{2}{|l|}{ Gleason Sum change } \\
\hline upgrade & $85(51.5)$ \\
\hline downgrade & $15(9.0)$ \\
\hline Clinically significant PCa (Epstein) & $158(95.8)$ \\
\hline \multicolumn{2}{|l|}{ D’Amico Classification } \\
\hline Low risk & $55(33.3)$ \\
\hline Intermediate risk & $61(37.0)$ \\
\hline High risk & $49(29.7)$ \\
\hline $\mathrm{N}+$ & $22(13.3)$ \\
\hline R+ & $36(21.8)$ \\
\hline L+ & 29 (17.6) \\
\hline V+ & $2(1.2)$ \\
\hline $\mathrm{Pn}+$ & $106(64.2)$ \\
\hline
\end{tabular}

Values expressed as median with range or number (\%). RPE, radical prostatectomy; $D R E$, digital rectal examination, PSAD, PSA density over PSA for more aggressive tumors with a $\mathrm{pGS} \geq 7$ $(\mathrm{p}=0.01)$. Moreover, sE-cadherin better detected PCas with a Gleason upgrade at RPE $(\mathrm{p}=0.01)$ than did serum PSA. Neither sE-cadherin, TIMP1, Galectin, Clusterin nor MMP9 correlated significantly with serum PSA.

\section{Discussion}

Estimating PCa malignancy in an era of personalized healthcare requires novel diagnostic and prognostic biomarkers to develop individual treatment strategies. Therefore, the potential of a panel of serum proteins to detect $\mathrm{PCa}$ and predict histopathological outcome in curatively treated disease was evaluated.

Out of 119 candidate molecules, sE-cadherin yielded the most promising potential to serve as a biomarker in the current study cohort with almost $96 \%$ clinically significant PCas according to the Epstein criteria [16]. E-cadherin, a type-I-cadherin, belongs to the cadherin family of transmembrane or membrane-associated glycoproteins located in the adherens junction and basolateral membrane in epithelial cells. It consists of a large extracellular domain, a transmembrane segment and a conserved cytoplasmic domain [18]. E-cadherin is indispensable in promoting cell-cell adhesion in a $\mathrm{Ca}^{2+}$-dependant manner and steers tissue morphogenesis [19]. During cancer progression, transcriptional E-cadherin reprogramming induces decreased adhesion and enhanced migration and invasion during the epithelial-to-mesenchymal transition of epithelial cells $[20,21]$. sE-cadherin is an $80-\mathrm{kDa}$ ectodomain of the full-length E-cadherin, which is proteolytically shed into the extracellular space through cleavage by secretases and caspases [18]. Concurrent to the disruption of adhesion junctions, EGFR and Wnt/ $\beta$-catenin pathway signaling, often involved in tumorigenesis, are activated [22]. Since E-cadherin cleavage has been linked to neoplastic adenoma-cancer progression, serum levels of sEcadherin have been shown to be augmented in patients with breast, gastric and colorectal cancer [23]. This is in line with our findings demonstrating that the serum concentration of sE-cadherin was elevated in patients with $\mathrm{PCa}$, more than other investigated candidates. Thanks to its stability in blood, sE-cadherin may be a suitable indicator for early PCa detection, due to accumulation during tumor-associated proteolysis [23].

So far, little has been reported about $\mathrm{sE}$-cadherin in connection with PCa. Ahmed et al. [24] investigated its potential as a biomarker in a group of 71 Egyptian males with PCa. In agreement with our findings, significant over-expression of sE-cadherin in the serum of tumor patients, compared to healthy individuals, was observed. No correlation with the clinical tumor stage was apparent. In contrast to our findings, the authors reported high expression of sE-cadherin, correlating with a poor Gleason grade and serum PSA. It is difficult to interpret their data 


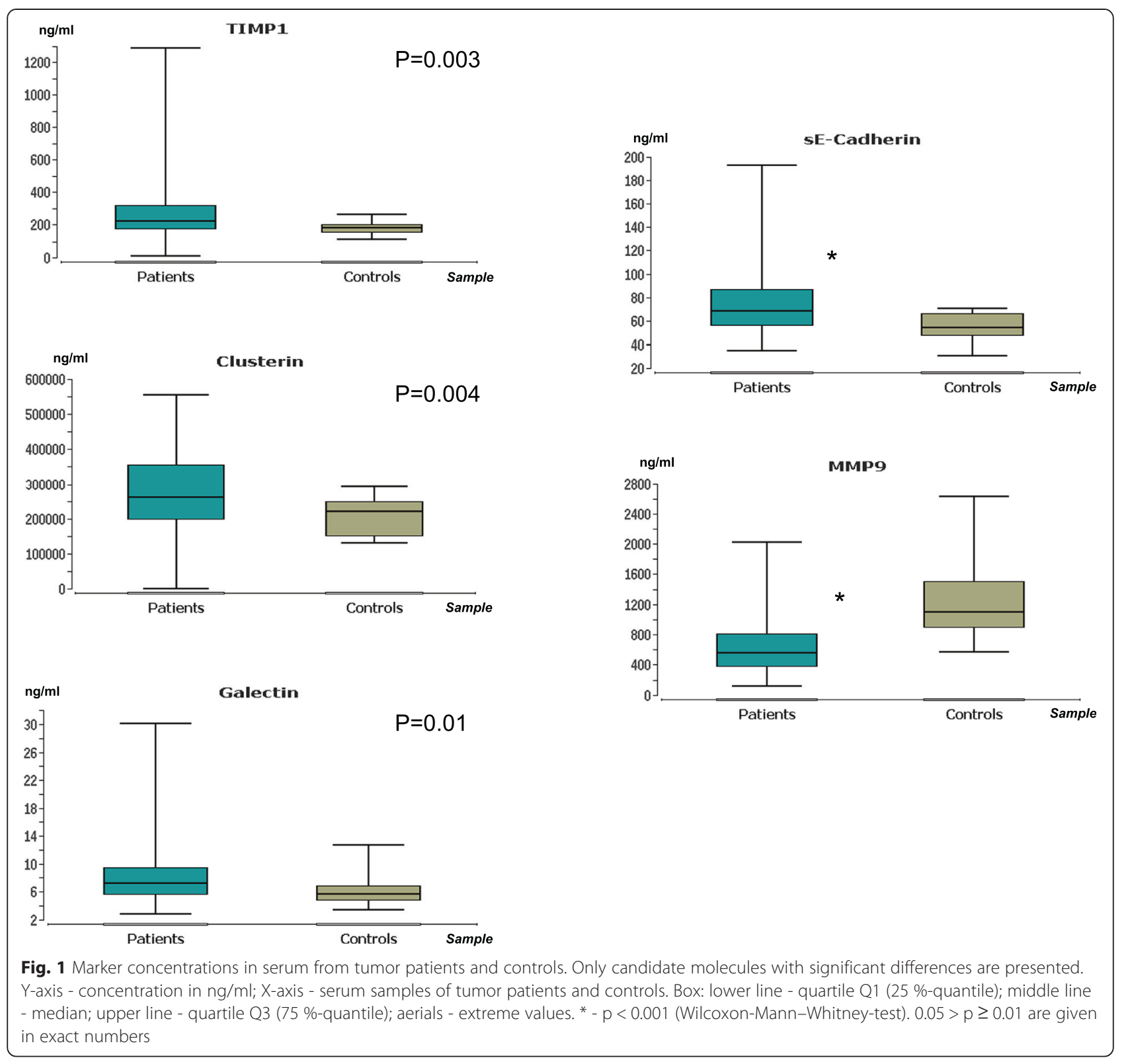

in the context of our findings due to the limited sample size of that cohort, median PSA of $70.7 \mathrm{ng} / \mathrm{ml}$ for tumor patients, most cancers classified as stage $\mathrm{D}$ and details about their staging procedure absent. These study characteristics harbor significant probability of undetected metastasis and therefore deviate from the present study population, which was clinically free from visceral- and

Table 3 Correlation of markers with prostatectomy Gleason Sum

\begin{tabular}{lrrrrr}
\hline & sE-Cadherin & MMP2 & MMP9 & TIMP1 & Clusterin \\
\hline p-value & 0.004599 & 0.020268 & 0.048087 & 0.027881 & 0.031574 \\
$\begin{array}{l}\text { Coefficient } \\
\text { (rho) }\end{array}$ & -0.219991 & -0.180763 & 0.154655 & 0.171867 & -0.168062 \\
\hline
\end{tabular}

bone-disease. Furthermore, alterations in Gleason grading procedures over time have resulted in significant tumor up-grading [25], making a study published in 1999 [24] difficult to compare with the present study carried out more than a decade later, due to bias stemming from the Will Rogers phenomenon. Later, Kuefer et al. [26] observed no difference between the expression of serum $\mathrm{sE-cadherin} \mathrm{in}$ 61 men with localized PCa compared to controls. On the contrary, a more contemporary series also involving 61 patients with clinically localized PCa, reported by Iacopino et al. [27], demonstrated serum sE-cadherin over-expression in PCa patients compared to that of healthy individuals, which is similar to our observation. However, no correlation of the marker with PSA, T-stage, GS, grade or 

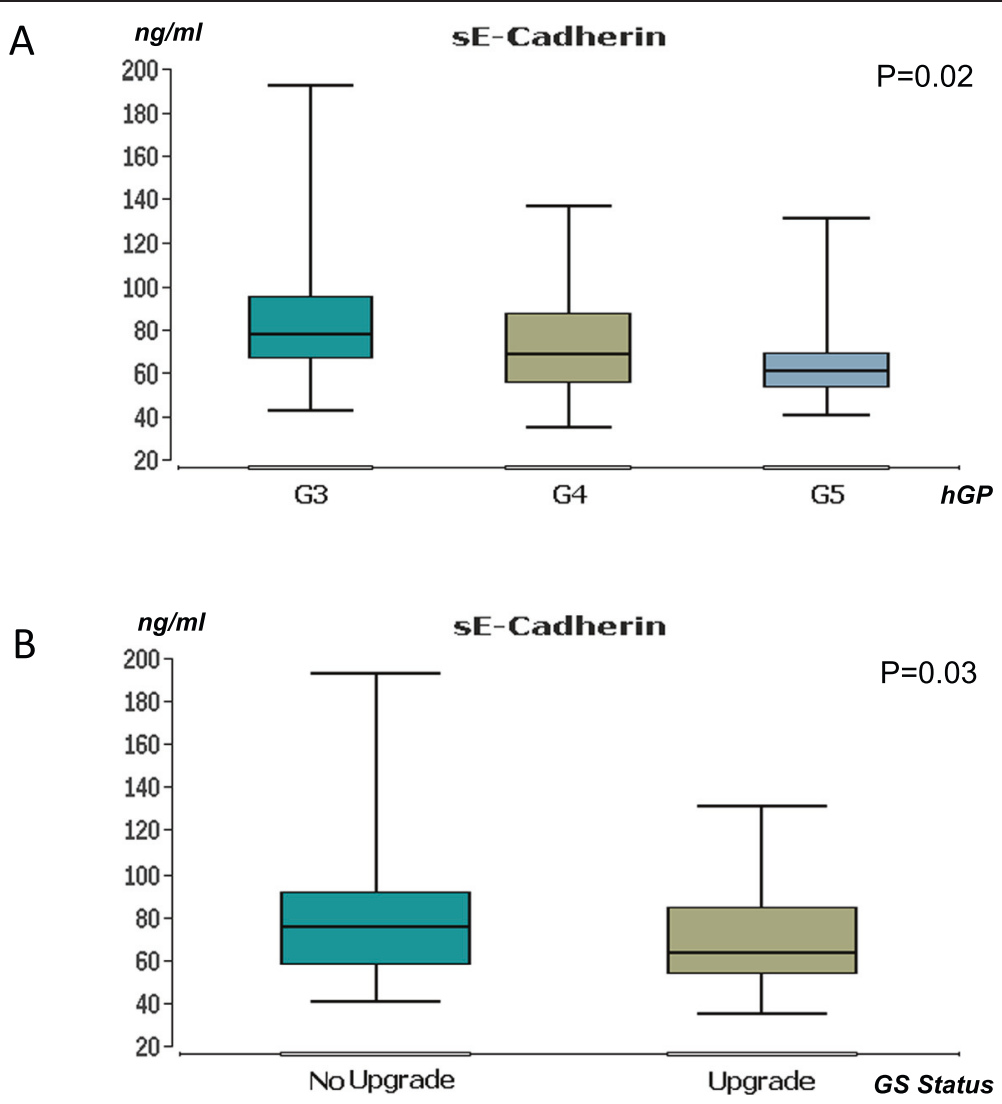

Fig. 2 Associations between sE-cadherin and histopathological parameters. a: Association between sE-cadherin expression and highest Gleason pattern. Y-axis - concentration in $\mathrm{ng} / \mathrm{ml}$; X-axis - serum samples of tumor patients distributed by the highest Gleason pattern (hGP). G3-G5 - Gleason pattern 3-5. P-value (Kruskal-Wallis-test). b: Association between sE-cadherin expression and Gleason sum upgrade. Y-axis - concentration in ng/ml; X-axis - serum samples of tumor patients distributed by upgrade status of the Gleason sum in prostatectomy specimen. P-value (Wilcoxon-Mann-Whitney-test). Box: lower line - quartile Q1 (25\%-quantile); middle line - median; upper line - quartile Q3 (75 \%-quantile); aerials - extreme values

R- and Pn-status was observed by those investigators. To best knowledge, the current assessment of sE-cadherin has taken place in the largest cohort of clinically nonmetastatic males with PCa. sE-cadherin correlated with the highest Gleason pattern, which is an important prognosis factor [28] and was also associated with a Gleason upgrade and pGS, which is more accurate than bGS at predicting biochemical recurrence after RPE [29]. Most importantly, for identifying more aggressive tumors with GS $\geq 7$ and tumors with a Gleason upgrade, sE-cadherin outmatched serum PSA without correlating with this marker.

The negative serum $\mathrm{SE}$-cadherin correlation with more aggressive $\mathrm{PCa}$ in this investigation requires further research since serum $\mathrm{sE}$-cadherin has been reported to rise with more aggressive or advanced stages in many other cancer types [23]. However, Juhasz et al. has provided evidence for significantly decreased expression of serum sE-cadherin in individuals with advanced and metastasized diffuse-type gastric cancer in contrast to intestinal cancer [30]. Careful analysis of cancer type as well as stage and grade specific molecular machinery pertaining to E-cadherin turnover is therefore warranted. Ongoing studies on SE-cadherin with respect to PCa should also take into consideration that proinflammatory cytokines [31], growth factors [32], as well as chemically induced oxidative stress [33] may contribute to its generation and possibly falsify results based on its expression.

Although sE-cadherin seems to be a promising biomarker, it may not be alone. Catenins, as well as CD44, are associated with E-cadherin in regulating prostate cancer cell adhesion [34]. In particular, CD44v8-10 have been shown to be negatively correlated with c-Myc expression, both in vitro and in vivo. The negative correlation may partly be attributable to redox stress-enhanced Wnt/betacatenin signaling [35].

The reason no distinct difference was apparent between the CD44 serum level in healthy subjects and tumor patients may have been because CD44 variants such as CD44v5, CD44v6 and CD44v10, possibly involved in prostate cancer progression, were not individually evaluated [36,37]. EpCAM differences between tumor patients and 
controls were also not apparent in the present investigation, which is in line with other reports [38]. EpCAM has been reported to be responsible for persistence of minimal residual disease and latent relapse of prostate cancer, presumably by affecting the susceptibility to the EGF ligand and regulating the AMPK signaling pathway [39]. Nevertheless, a prognostic/diagnostic role of EpCAM in EGFR activated prostate cancer cells should not be ruled out.

The same ambivalence might apply to MMP9. Although this protein has been suggested to correlate with prostate cancer progression, the correlation has not always been substantiated. Recent experiments documented that MMP9 might even exert tumor-suppressive properties. In fact, elevated MMP9 secretion has been shown to result in decreased prostate cancer cell proliferation, coupled to increased sE-cadherin shedding [40]. The combined effect might explain the inverse correlation between sE-cadherin and MMP9 found in the present investigation, whereby reduced MMP9 and elevated sE-cadherin may drive tumor proliferation forward. Still, this is hypothetical and requires further evaluation.

The present investigation is limited by its single-center design and lack of external validation. To account for regional and racial disparity regarding the biological characteristics of PCa [41], validation studies with patient cohorts from other centers would be desirable. The potential of sE-cadherin as a biomarker could thereby be more exactly appraised.

\section{Conclusions}

To sum up, sE-cadherin performed best out of a large panel of serum proteins in terms of diagnostic and predictive potential in 165 patients with clinically localized PCa. Therefore, this molecule merits further investigation as a biomarker for $\mathrm{PCa}$.

\section{Competing interests}

The authors declare that they have no competing interests.

\section{Authors' contributions \\ IT: study conception, data analysis, statistics and manuscript writing. KT: investigations, statistics. EJ: investigations. KMG: data interpretation, manuscript revision. HB: data interpretation, manuscript revision. RM: data interpretation, statistics. GB: study conception, data analysis. EO: investigations. HA: statistics. KN: manuscript revision, data analysis. AH: study conception, data analysis, statistics and manuscript revision. RAB: study conception, data analysis, statistics and manuscript revision. All authors read and approved the final manuscript.}

\section{Authors' information}

Axel Haferkamp and Roman A Blaheta contributed equally as senior authors.

\section{Acknowledgments}

This work was supported by the Dr. Illing Stiftung, Hans und Wolfgang Schleussner-Stiftung and Banss Stiftung.

\footnotetext{
Author details

'Department of Urology, Goethe-University, Frankfurt am Main, Germany. ${ }^{2}$ Department of Surgery, Goethe-University, Frankfurt am Main, Germany. ${ }^{3}$ Institute for Biostatistics, Goethe-University, Frankfurt am Main, Germany. ${ }^{4}$ Department of Vascular and Endovascular Surgery, Goethe-University, Frankfurt am Main, Germany.
}

Received: 16 February 2015 Accepted: 1 May 2015

Published online: 14 May 2015

\section{References}

1. Shafique K, Oliphant R, Morrison DS. The impact of socio-economic circumstances on overall and grade-specific prostate cancer incidence: a population-based study. Br J Cancer. 2012;107:575-82.

2. Roehrborn CG, Black LK. The economic burden of prostate cancer. BJU Int. 2011;108:806-13.

3. Joniau S, Pfister D, de la Taille A, Gaboardi F, Thompson A, Ribal MJ. Controversies on individualized prostate cancer care: gaps in current practice. Ther Adv Urol. 2013;5:233-44.

4. Adami HO. The prostate cancer pseudo-epidemic. Acta Oncol. 2010;49:298-304

5. Heidenreich A, Bastian PJ, Bellmunt J, Bolla M, Joniau S, van der Kwast T, et al. EAU guidelines on prostate cancer. part 1: screening, diagnosis, and local treatment with curative intent-update 2013. Eur Urol. 2014;65:124-37.

6. Caras RJ, Sterbis JR. Prostate cancer nomograms: a review of their use in cancer detection and treatment. Curr Urol Rep. 2014;15:391.

7. Freedland S, Mithal P. Editorial comment. Urology. 2013;81:1208.

8. Tsaur I, Noack A, Makarevic J, Oppermann E, Waaga-Gasser AM, Gasser M et al. CCL2 Chemokine as a Potential Biomarker for Prostate Cancer: A Pilot Study. Cancer Res Treat. 2014; doi:10.4143/crt.2014.015

9. Dijkstra S, Birker IL, Smit FP, Leyten GH, de Reijke TM, van Oort IM, et al. Prostate cancer biomarker profiles in urinary sediments and exosomes. J Urol. 2014;191:1132-8.

10. Amaro A, Esposito Al, Gallina A, Nees M, Angelini G, Albini A, et al. Validation of proposed prostate cancer biomarkers with gene expression data: a long road to travel. Cancer Metastasis Rev. 2014;33:657-71.

11. Edge SB. American joint committee on cancer., american cancer society., Teton data systems (firm). AJCC cancer staging handbook from the AJCC cancer staging manual. 7th ed. New York: Springer; 2010.

12. Wittekind C. International union against cancer. TNM-klassifikation maligner tumoren. 6. Aufl., korrig. Nachdr. ed. Heidelberg: Springer Medizin; 2005.

13. Epstein Jl, Allsbrook Jr WC, Amin MB, Egevad LL, Committee IG. The 2005 International Society of Urological Pathology (ISUP) consensus conference on gleason grading of prostatic carcinoma. Am J Surg Pathol. 2005;29:1228-42

14. D'Amico AV, Whittington R, Malkowicz SB, Schultz D, Blank K, Broderick GA, et al. Biochemical outcome after radical prostatectomy, external beam radiation therapy, or interstitial radiation therapy for clinically localized prostate cancer. JAMA. 1998;280:969-74.

15. Heidenreich A, Aus G, Bolla M, Joniau S, Matveev VB, Schmid HP, et al. EAU guidelines on prostate cancer. Eur Urol. 2008;53:68-80.

16. Leyten GH, Hessels D, Jannink SA, Smit FP, de Jong H, Cornel EB, et al. Prospective multicentre evaluation of PCA3 and TMPRSS2-ERG gene fusions as diagnostic and prognostic urinary biomarkers for prostate cancer. Eur Urol. 2014;65:534-42.

17. Vittinghoff $E$, McCulloch CE. Relaxing the rule of ten events per variable in logistic and Cox regression. Am J Epidemiol. 2007;165:710-8.

18. David JM, Rajasekaran AK. Dishonorable discharge: the oncogenic roles of cleaved E-cadherin fragments. Cancer Res. 2012;72:2917-23.

19. Halbleib JM, Nelson WJ. Cadherins in development: cell adhesion, sorting, and tissue morphogenesis. Genes Dev. 2006;20:3199-214.

20. van Roy F, Berx G. The cell-cell adhesion molecule E-cadherin. Cell Mol Life Sci. 2008;65:3756-88

21. Gheldof A, Berx G. Cadherins and epithelial-to-mesenchymal transition. Prog Mol Biol Transl Sci. 2013;116:317-36

22. De Freitas Silva BS, Yamamoto-Silva FP, Pontes HA, Pinto Junior Ddos S. E-cadherin downregulation and Twist overexpression since early stages of oral carcinogenesis. J Oral Pathol Med. 2014;43:125-31.

23. Repetto O, De Paoli P, De Re V, Canzonieri V, Cannizzaro R. Levels of soluble E-cadherin in breast, gastric, and colorectal cancers. Biomed Res Int. 2014;2014:408047.

24. Ahmed Ml, Abd-Elmotelib F, Farag RM, Ziada NA, Khalifa A. Evaluation of some tissue and serum biomarkers in prostatic carcinoma among Egyptian males. Clin Biochem. 1999:32:439-45.

25. Danneman D, Drevin L, Robinson D, Stattin P, Egevad L. Gleason inflation 1998-2011: a registry study of 97168 men. BJU Int. 2015;115:248-55.

26. Kuefer R, Hofer MD, Gschwend JE, Pienta KJ, Sanda MG, Chinnaiyan AM, et al. The role of an $80 \mathrm{kDa}$ fragment of E-cadherin in the metastatic progression of prostate cancer. Clin Cancer Res. 2003;9:6447-52. 
27. lacopino F, Pinto F, Bertaccini A, Calarco A, Proietti G, Totaro A, et al. Soluble E-cadherin and IL-6 serum levels in patients affected by prostate cancer before and after prostatectomy. Oncol Rep. 2012;28:370-4.

28. Epstein JI. An update of the Gleason grading system. J Urol. 2010;183:433-40.

29. D'Amico AV, Whittington R, Malkowicz SB, Schultz D, Schnall M, Tomaszewski JE, et al. A multivariate analysis of clinical and pathological factors that predict for prostate specific antigen failure after radical prostatectomy for prostate cancer. J Urol. 1995:154:131-8.

30. Juhasz M, Ebert MP, Schulz HU, Rocken C, Molnar B, Tulassay Z, et al. Dual role of serum soluble E-cadherin as a biological marker of metastatic development in gastric cancer. Scand J Gastroenterol. 2003;38:850-5.

31. Grabowska MM, Day ML. Soluble E-cadherin: more than a symptom of disease. Front Biosci (Landmark Ed). 2012;17:1948-64.

32. Gumbiner BM. Regulation of cadherin adhesive activity. J Cell Biol. 2000;148:399-404.

33. Parrish AR, Catania JM, Orozco J, Gandolfi AJ. Chemically induced oxidative stress disrupts the E-cadherin/catenin cell adhesion complex. Toxicol Sci. 1999;51:80-6.

34. Kallakury BV, Sheehan CE, Ross JS. Co-downregulation of cell adhesion proteins alpha- and beta-catenins, p120CTN, E-cadherin, and CD44 in prostatic adenocarcinomas. Hum Pathol. 2001;32:849-55.

35. Yoshida GJ, Saya H. Inversed relationship between CD44 variant and c-Myc due to oxidative stress-induced canonical Wnt activation. Biochem Biophys Res Commun. 2014;443:622-7.

36. Lein M, Jung K, Weiss S, Schnorr D, Loening SA. Soluble CD44 variants in the serum of patients with urological malignancies. Oncology. 1997;54:226-30.

37. Tei H, Miyake H, Harada K, Fujisawa M. Expression profile of CD44s, CD44v6, and CD44v10 in localized prostate cancer: effect on prognostic outcomes following radical prostatectomy. Urol Oncol. 2014;32:694-700.

38. Rupp AK, Rupp C, Keller S, Brase JC, Ehehalt R, Fogel M, et al. Loss of EpCAM expression in breast cancer derived serum exosomes: role of proteolytic cleavage. Gynecol Oncol. 2011;122:437-46.

39. Yoshida GJ, Saya H. EpCAM expression in the prostate cancer makes the difference in the response to growth factors. Biochem Biophys Res Commun. 2014;443:239-45.

40. Biswas MH, Du C, Zhang C, Straubhaar J, Languino LR, Balaji KC. Protein kinase D1 inhibits cell proliferation through matrix metalloproteinase-2 and matrix metalloproteinase-9 secretion in prostate cancer. Cancer Res. 2010;70:2095-104.

41. Zhang H, Messing EM, Travis LB, Hyrien O, Chen R, Milano MT, et al. Age and Racial Differences among PSA-Detected (AJCC Stage T1CNOMO) Prostate Cancer in the U.S.: A Population-Based Study of 70,345 Men. Front Oncol. 2013;3:312.

\section{Submit your next manuscript to BioMed Central and take full advantage of:}

- Convenient online submission

- Thorough peer review

- No space constraints or color figure charges

- Immediate publication on acceptance

- Inclusion in PubMed, CAS, Scopus and Google Scholar

- Research which is freely available for redistribution 